\title{
A Study on the Strategies of Developing the Autonomous Human Social Behavior of Virtual Users Based on Psychological Interpretation in an Atypical Architectural Shape
}

\author{
Young Lim Lee1), Yun Gil Lee $\left.{ }^{2}\right)$
}

\begin{abstract}
Virtual user's autonomous behavior is helpful in stances when the architects ignore human influences, which is one of the most significant factors to evaluate the value of buildings in the process of architectural design. This paper aims to articulate the strategies of developing the autonomous human social behavior of virtual users based on psychological interpretation in an atypical architectural building. First, we established the intellectual triggers of user actions in atypical architectural architecture that are crucial to the creation of a user action simulation framework. Human communicate both internally with the form of a structure and socially with those inside an atypical setting. In order to lay the groundwork for computerization, it is important to understand both the type of collective action being taken in an atypical environment and the origin of that form. Second, we intended to computerize the ideas of "behavioral conformity," "in-group bias," and "behavior setting," which are representative cases of social behaviors in atypical architecture. In consideration of the characteristics of each behavioral type, a technical method for simulation was presented. In the results of this study, strategies of developing the autonomous social behavior of virtual users are suggested. They will then be used as a basis for the advancement of technologies that can be incorporated into the existing ActoViz framework to provide a more proper simulation for user actions.
\end{abstract}

Keywords: Virtual User, Autonomous Behavior, Human Social Behavior, Psychological Interpretation, Atypical Architectural Shape, Affordance

\section{Introduction}

Atypically designed buildings are becoming increasingly prevalent in contemporary society, and the architect's capacity to formulate atypical structures is essential. The construction method of an atypical construction varies somewhat from that of conventional architecture in that it

Received(June 16, 2020), Review Result(1st: August 8, 2020, 2nd: September 24, 2020), Accepted(October 28, 2020)

1) (Professor) 16890 Department of Psychology \& Psychotherapy, Dankook University, 119 Dandae-ro Dongnam-gu, Cheonan-si, Chungcheongnam-do, Republic of Korea

email: younglee13@dankook.ac.kr

2) (Professor, Corresponding Author) 31499 Department of Architecture, Hoseo University, 20 Hoseo-ro 79 beon-gil, Baebang-eup, Asan-si, Chungcheongnam-do, Republic of Korea

email: yglee@hoseo.edu 
A Study on the Strategies of Developing the Autonomaus Human Social Behavior of Virtual Users Based on Psychological Interpretation in an Atypical Architectural Shape

involves an equally uncommon type of design. However when planning an atypical structure, the architect may neglect the comfort and welfare of customers, which are usually important criteria for determining the importance of buildings. Thus, to avoid the protection and comforts of consumers from being ignored, the use of human behavior modeling is integrated into the design sequence of constructing atypical conditions.

The goal of this paper is to propose techniques for the creation of an autonomous human social activity of virtual users based on psychological comprehension in atypical architectural features. In the first instance, the authors examined the intellectual reasons of human social activity in atypical structure that are a basis to the development of a human behavior simulation framework. Users participate both internally in the layout of a structure and externally with others inside an curved environment, and it is crucial to understand both the type of social interaction taking place in an atypical atmosphere and the cause for this method in order to ready the ground for computerization. Second, we intended to computerize the ideas of "behavioral conformity," "in-group bias," and "behavior setting," which are representative cases of social behaviors in atypical buildings. In consideration of the characteristics of each behavioral type, a technical method for simulation was presented. The findings from this study should be used as a basis for the advancement of technologies that can be incorporated into the previously existing ActoViz framework for a more realistic evaluation of user behavior[1][2].

\section{Atypical Building Design and Human Social Behavior Simulation}

Atypical architecture may reflect non-Euclidean building forms rather than traditional modern Euclidean shapes. They are often assembled of rounded features that are difficult to see in modernist design, as seen in [Fig. 1]. When constructing atypical structures, architects can quickly skip human factors when they typically concentrate more on the irregularly shaped of structures. Human behavior modeling technique would also enable designers to recognize human-related problems in the design manner. Hong and Lee stated that the portrayal of human actions in atypical design has a beneficial impact as it enhances architectural problem-finding skills, associated design growth, current design area and non-common design[3-5]. In the previous research, we looked at the implementation of agent-based simulation technology in the context of an individual behaving in corresponding to an atypical house. However the conduct of the user was decided to be affected not only by the physical context of the atypical structure, but also by the social settings. In order to create a optimal framework of behavior simulation, the aim of this research was to examine the social behavior of users in atypical 
architecture and to classify their intellectual triggers[6-8].

\section{Psychological Analysis of Human Social Behaviors in Atypical Architecture}

In order to identify the psychological causes of users' social behavior in atypical structures, we investigated and analyzed user behaviors in atypical spaces. The researchers toured symbolic atypical buildings to analyze user behavior and categorize common activities inside atypical buildings. The analysis of the relevant examples using psychological theories proceeded as follows:

\subsection{Self-Perception}

The "self-perception theory" proposed by Daryl Bem in 1972 suggests that when we take the observer's position when we are not sure of our own attitude[9][10]. That is to say, observing our behavior and the environment around us when our attitude is weak or uncertain results in our inferring our attitudes as if others were observing us. Among the behaviors observed in this study, users were seen sitting on a chair or staircase and leaning against a pillar. It is possible that an individual may sit or lean for the purpose of rest, but the behaviors can be explained by a psychosocial point of view. From the self-perception theory, a person may continue to rest when leaning or sitting somewhere because he or she perceives his or her own behavior and infers "Oh, I need a rest now". The theory that one's behavior might be modified by self-perception can be interpreted by the "facial feedback effect" in which our feelings are affected by imitating the gestures or acts of others. In fact, when we observe others' faces, postures, or voices, we naturally and unconsciously imitate our actions to match those of others. This is because matching behaviors with others allows us to reconcile our feelings with theirs[11]. In a similar way, as we see people leaning against walls or sitting on stairs and benches, we unwittingly mimic our actions to match their behavior and other emotional states.

\subsection{Behavioral Conformity}

[Fig. 1] shows the examples of behavioral conformity. Since humans live in a society, our behaviors are influenced by the presence of others. One of the effects of others' presence is conformity. Conformity is not simply a matter of imitating other's behaviors but rather involves 
A Study on the Strategies of Developing the Autonomaus Human Social Behavior of Virtual Users Based on Psychological Interpretation in an Atypical Architectural Shape

following their inferred beliefs and behaviors. We are influenced by others' behaviors no matter if we are superficially acknowledging or actually conforming to them. Seeing that most people lean against a wall, relax or sit on a chair can be perceived as behaviors that are in harmony with others. There behaviors can be perceived as conformity without conscious intent. However, these behaviors can be explained as a deliberate conformity or cooperative activity with the group due to compliance with one's own party.

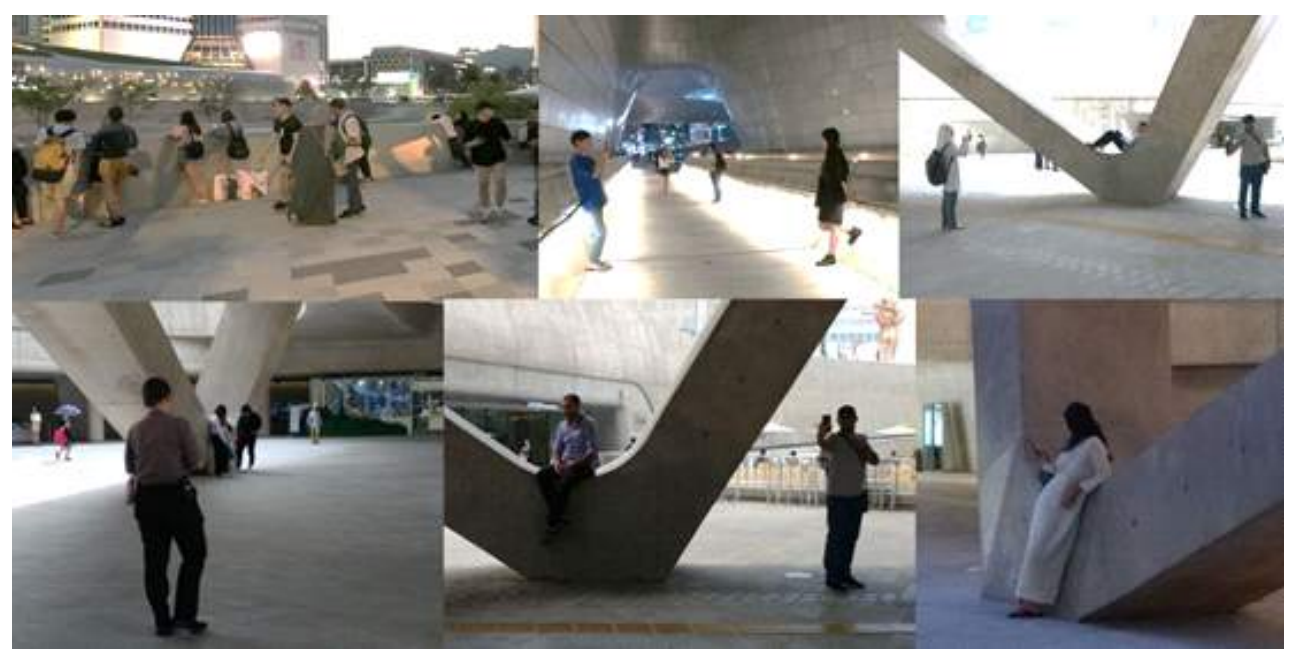

[Fig. 1] Examples of Human Social Behaviors Related to Behavioral Conformity in Atypical Buildings

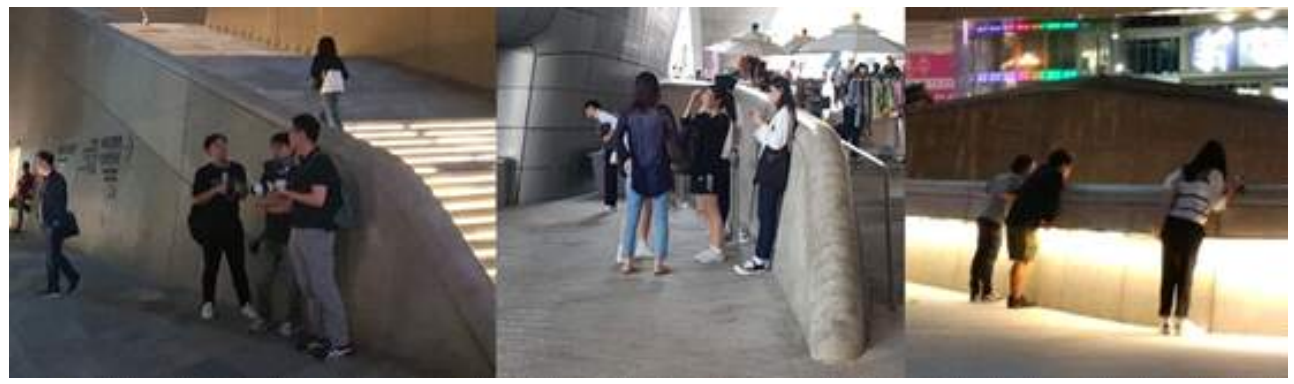

[Fig. 2] Examples of Human Social Behaviors Related to In-group Bias in Atypical Buildings

\subsection{In-Group Bias}

[Fig. 3] shows examples of in-group bias in the community. It will seem normal for people to sit on it for the purpose of a chair. However no one has odd habits, such as laying on a chair, climbing a chair, or carrying a chair while most of them are seated. This is because humans have a desire to belong to a community and build relationships in society. In other 
words, we do not want to leave our own group by acting unusually because of "in-group bias" referred as a tendency to conform to the group we belong to. Therefore, by acting in the same way as the majority, we will feel that we belong to in-group, whether consciously or not, and are supported by close and intimate relationships.

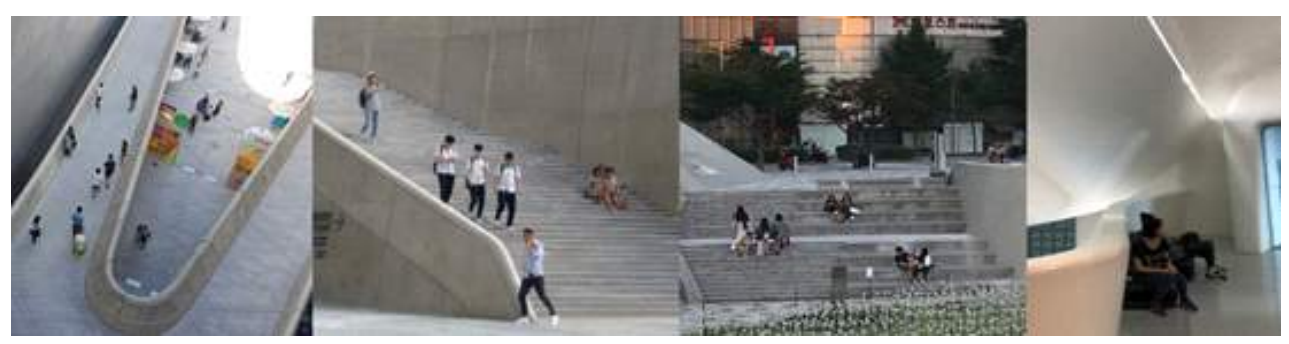

[Fig. 3] Examples of Human Social Behaviors Related to Behavior Setting in Atypical Building

\subsection{Behavior Setting}

People's behaviors are shaped by the group to which they belong, but it is important to note that environment also has a role because group can be shaped by the situations the group encountered([Fig. 3]). Individuals and communities for which they identify reside within the physical world situated at a given site, and these locations inevitably influence the dynamics of the community and the outcomes. The atmosphere is often a physical area that require one to behave in compliance with the desire of the context, and is considered a behavioral setting. Ordering lines in food restaurants, waiting places in hospitals, lecture rooms in universities, meeting rooms in companies, and chairs in parks can all be considered behavioral situations. The reason for this is that human behavior in these place is not their personal characteristics, but rather the characteristics of the space. When you enter a fast food restaurant, for example, people line up, order food, pay for food, find seats and sit down. Ecological psychologist Roger Barker has indicated that certain acts (at least those that are normal and routine) are decided by the conditions in which they arise. Behavioral situations imply unique spaces in which the members of the group communicate. For instance, people use the objects that make up the situation in a very predictable and regular way, which is similar to sitting in a chair when people enter a room having chairs. There are also cases where people and places themselves fit together without any problems. Barker used the term "synomorphy" to explain the degree of correspondence between human and context. If the level of synomorphy is high, people can respond well to the physical world and make proper use of the elements of the environment. The physical world, such as stairs or tables, also demonstrates a strong degree of 
A Study on the Strategies of Developing the Autonomaus Human Social Behavior of Virtual Users Based on Psychological Interpretation in an Atypical Architectural Shape

synomorphism with the action of walking down or sitting down[9][10].

\section{Strategies for Developing the Autonomous Social Behavior of a Virtual User}

This section introduces how to computerize behavioral conformity, in-group bias, and behavior setting among four users' social interactions in an atypical architectural space. In other words, this can be interpreted as a strategy to develop the automated social interaction of agents. Because self-perception is a psychological change in the user that is not revealed to the outside world, it is difficult to computerize. Thus, it is hard to provide appropriate feedback to the architect in this case because self-perception is not visible[12].

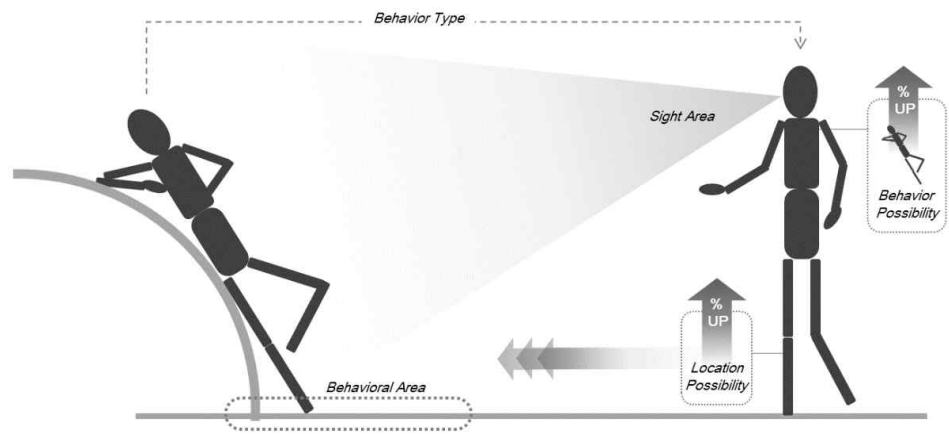

[Fig. 4] Strategy to Computerize 'Behavioral Conformity' in a Human Behavior Simulation

\subsection{Development Strategy for Behavioral Conformity}

[Fig. 4] shows the strategy for computerizing behavioral conformity. Behavioral conformity consists of social action that conforms to the behavior of another party. In other words, it involves the observation of another person's behavior and the modeling of it in a similar situation. Computationally, when another agent's behavior comes into the agent's field of view, the probability of similar behavior increases and is likely to develop into behavioral conformity. As the simulation progresses, a phenomenon may occur in which the number of agents enacting similar behavior increases, which was similar to the behavior of users found through the observation of field survey. 


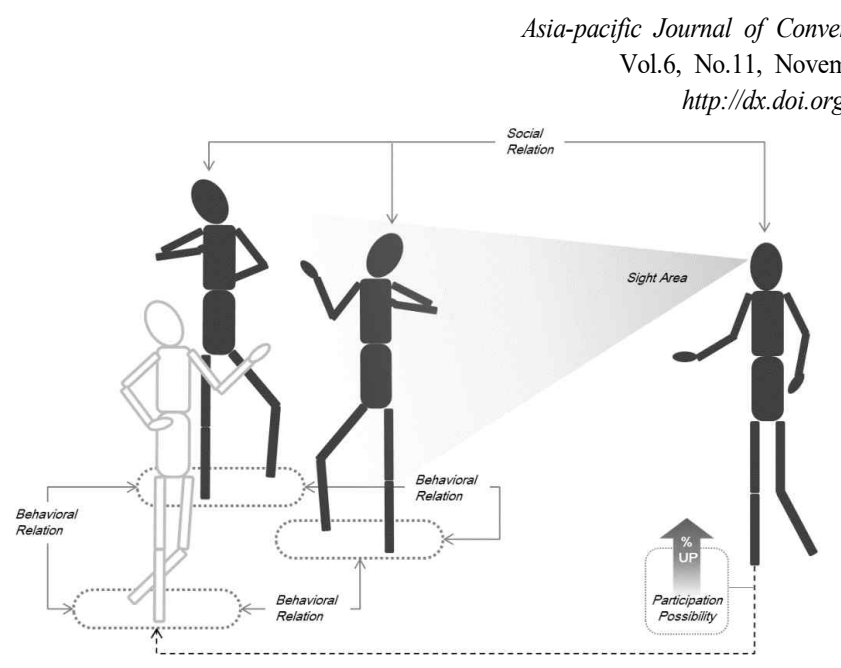

[Fig. 5] Strategy to Computerize 'In-group Bias' in a Human Behavior Simulation

\subsection{Development Strategy for In-Group Bias}

In-group bias emerges as a result of social follow-up. In other words, people with social relationships take actions that are similar or related to another person's actions. In order to develop social interaction in this way, it is a priority to establish the social relations of agents. When other socially related agents enter the field of view, in-group bias can be implemented in a way that increases the likelihood of causing socially relevant actions in preference to other actions. [Fig. 5] illustrate the strategy to computerize this idea.

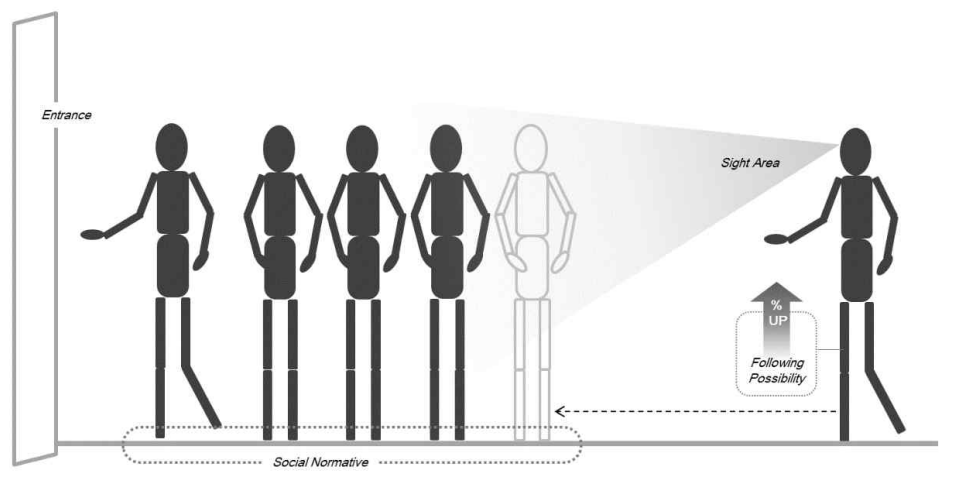

[Fig. 6] Strategy to Computerize In-group Bias in a Human Behavior Simulation

\subsection{Development Strategy for Behavior Setting}

Social interactions related to behavior setting should be considered in two ways. First, social 
A Study on the Strategies of Developing the Autonomaus Human Social Behavior of Virtual Users Based on Psychological Interpretation in an Atypical Architectural Shape

interaction can be expressed through the social "normativity" of the physical space. As shown in [Fig. 6], it is a social promise to stand in line when entering the entrance. Social interactions can be implemented in such a way that events are set up in physical situations where social promises are expected, and agents are subject to specific rules in this location.

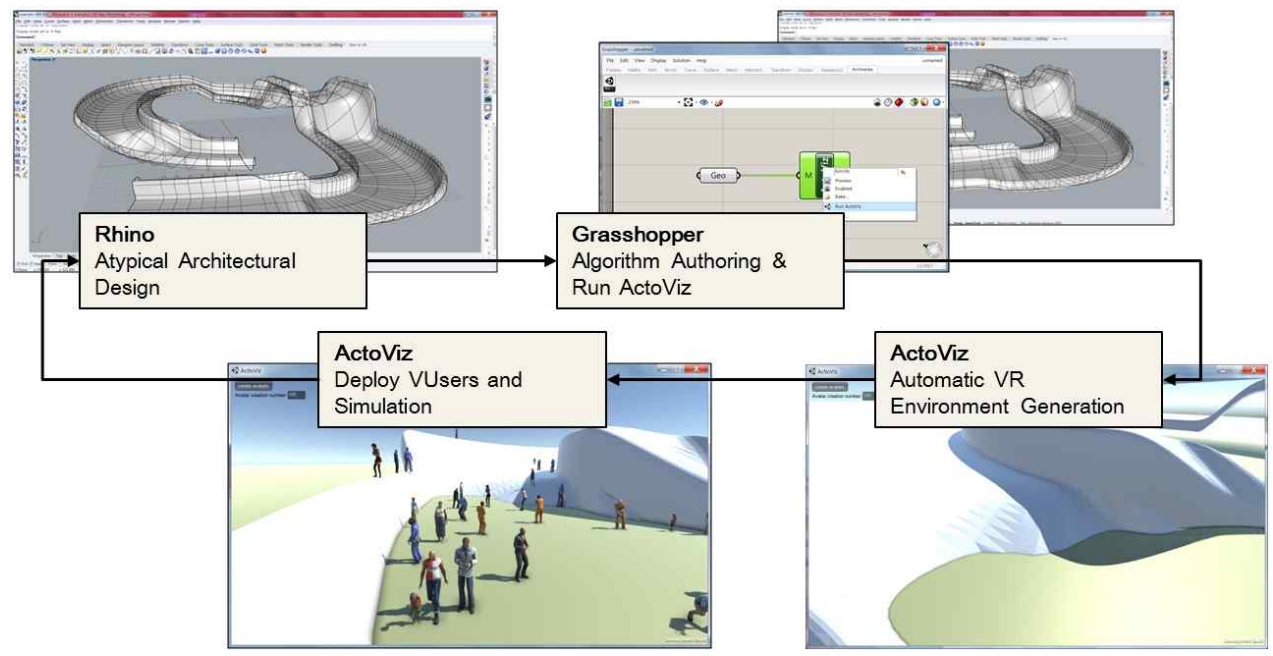

[Fig. 7] Process of Executing ActoViz using Rhino and Grasshopper[7]

\section{Conclusion and Discussion}

This study introduced a strategy for the computational realization of social interaction in an atypical space. Ultimately, this is a study designed to facilitate the development of tools that perform user simulations in the process of constructing an atypical design. In other words, the purpose is to show how it is possible to present the realities of social interaction within the designed space to the architect by visualizing the social interaction of an agent in the form of a person in real-time. Specifically, we focused on establishing a strategy for development and described it in some detail. It is now necessary to computerize the methods presented in this study through future studies. [Fig. 7] shows the process of executing ActoViz which has been developed for human behavior simulation in an atypical design process. When designing atypical built environment and outer wall prototype design utilizing Rhino and Grasshopper functions, the designed ActoViz module can be put in Grasshopper and connected to proposed algorithm to build a simulation framework. As the simulation is run on the ActoViz system, it delivers the modeled atypical space to Unity3D to create simulated 3D space in real time. 
Independent characters are installed in simulated spaces and operate separately on the atypical architectural board. The behavioral representation of autonomous characters can have an effect on the architects' decision-making process. In a further review, the techniques built in this paper will be extended to ActoViz[7][13]. In addition, a more in-depth discussion is required on the relationship between atypical space and social interaction.

\section{Acknowledgement}

This work was supported by the National Research Foundation of Korea (NRF) grant funded by the Korean government (MSIT) (NRF-2018R1A2B6005827).

\section{References}

[1] H. Oh, Impact of Metacognition on Simulation Effectiveness in Problem-based Learning using Simulation, International Journal of Computer Science and Information Technology for Education, (2016), Vol.1, No.1, pp.61-66.

[2] J. Tae, The effect of Design Thinking - based STEAM education on elementary school student interest in math and science, personality, and science and technology career choice, Asia-Pacific Journal of Educational Management Research, (2016), Vol.1, No.1, pp.1-6.

[3] S. Hong, Y. Lee, The effects of human behavior simulation on architectural major students' fire egress planning, Journal of Asian Architecture and Building Engineering, (2018), Vol.17, No.1, pp.125-132., DOI: 10.3130/jaabe.17.125

[4] Y. Lee, A study on the effect of human factor for atypical design in the architectural design studio, Proceedings of Human Systems Engineering and Design (IHSED 2018), Vol.876, (2018), October 25-27; France, DOI: https://doi.org/10.1007/978-3-030-02053-8_21

[5] S. Hong, D. Schaumann, Y. E. Kalay, Human behavior simulation in architectural design projects: An observational study in an academic course, Computer, Environment, and Urban Systems, (2016), Vol.60, pp.1-11, DOI: 10.1016/j.compenvurbsys.2016.07.005

[6] D. Simeone, Y. E. Kalay, D. Schaumann, S. Hong, Modeling and simulating use processes in buildings, Proceedings of the 31st international conference on education and research in computer aided architectural design in Europe, pp.59-66, (2013), September 18-20; Delft, Netherlands.

[7] Y. Lee, ActoViz: A human behavior simulator for the evaluation of the dwelling performance of an atypical architectural space, HCI International 2019 - Posters, Vol.1034, (2019), July 26-31; FL, USA, DOI: https://doi.org/10.1007/978-3-030-23525-3_48

[8] Won-Geun So, Ha-Kyun Kim, An Empirical Study on the Effect of the Social Norms on Assault Behavior through Cyber Bullying, Asia-pacific Journal of Convergent Research Interchange, (2020), Vol.6, No.2, pp.149-158, DOI: 10.21742 /apjcri.2020.02.14 
A Study on the Strategies of Developing the Autonomaus Human Social Behavior of Virtual Users Based on Psychological Interpretation in an Atypical Architectural Shape

[9] D. G. Myers, Social Psychology, 11th edition. McGraw-Hill Education, (2012)

[10] D. R. Forsyth, Group dynamics, 5th edition, Wadsworth Cengage Learning, (2014)

[11] E. Hatfield, J. T. Cacioppo, R. L. Rapson, Primitive emotional contagion, Review of personality and social psychology, (1992), Vol14, pp.151-177.

[12] Seong-Ju Oh, Ha-Sung Kong, The Strategies to Supply Efficient Fire fighting Force in High-rise Building by NFPA 550 Guide to the Fire Safety Concepts Tree: Focusing on Automatic Fire Suppression, Asia-pacific Journal of Convergent Research Interchange, (2020), Vol.6, No.4, pp.67-80, DOI: 10.21742/apjcri.2020.04.07

[13] G. Goldschmidt, Linkography, Cambridge, MIT Press, (2014) 